\title{
Population genetic structure and natural selection of apical membrane antigen-1 in Plasmodium vivax Korean isolates
}

Jung-Mi Kang ${ }^{1 \dagger}$, Jinyoung Lee ${ }^{1 \dagger}$, Pyo-Yun Cho², Sung-Ung Moon ${ }^{3}$, Hye-Lim Ju', Seong Kyu Ahn², Woon-Mok Sohn ${ }^{1}$, Hyeong-Woo Lee ${ }^{4}$, Tong-Soo Kim² ${ }^{2^{*}}$ and Byoung-Kuk Na ${ }^{1^{*}}$

\begin{abstract}
Background: Plasmodium vivax apical membrane antigen-1 (PVAMA-1) is a leading candidate antigen for blood stage malaria vaccine. However, antigenic variation is a major obstacle in the development of an effective vaccine based on this antigen. In this study, the genetic structure and the effect of natural selection of PvAMA-1 among Korean P. vivax isolates were analysed.
\end{abstract}

Methods: Blood samples were collected from 66 Korean patients with vivax malaria. The entire PvAMA-1 gene was amplified by polymerase chain reaction and cloned into a TA cloning vector. The PVAMA-1 sequence of each isolate was sequenced and the polymorphic characteristics and effect of natural selection were analysed using the DNASTAR, MEGA4, and DnaSP programs.

Results: Thirty haplotypes of PvAMA-1, which were further classified into seven different clusters, were identified in the 66 Korean P. vivax isolates. Domain II was highly conserved among the sequences, but substantial nucleotide diversity was observed in domains I and III. The difference between the rates of non-synonymous and synonymous mutations suggested that the gene has evolved under natural selection. No strong evidence indicating balancing or positive selection on PVAMA-1 was identified. Recombination may also play a role in the resulting genetic diversity of PVAMA-1.

Conclusions: This study is the first comprehensive analysis of nucleotide diversity across the entire PVAMA-1 gene using a single population sample from Korea. Korean PVAMA-1 had limited genetic diversity compared to PvAMA-1 in global isolates. The overall pattern of genetic polymorphism of Korean PVAMA-1 differed from other global isolates and novel amino acid changes were also identified in Korean PVAMA-1. Evidences for natural selection and recombination event were observed, which is likely to play an important role in generating genetic diversity across the PVAMA-1. These results provide useful information for the understanding the population structure of $P$. vivax circulating in Korea and have important implications for the design of a vaccine incorporating PVAMA-1.

Keywords: Plasmodium vivax, Apical membrane antigen-1, Vaccine, Genetic polymorphism, Natural selection, Korea

\footnotetext{
*Correspondence: tongsookim@inha.ac.kr; bkna@gnu.ac.kr

†Jung-Mi Kang and Jinyoung Lee are equally contributed to this study

${ }^{1}$ Department of Parasitology and Tropical Medicine, Institute of Health

Sciences, Gyeongsang National University School of Medicine,

Jinju 660-751, Republic of Korea

${ }^{2}$ Department of Tropical Medicine, Inha Research Institute for Medical

Sciences, Inha University School of Medicine, Incheon 400-712,

Republic of Korea

Full list of author information is available at the end of the article
} 


\section{Background}

Plasmodium vivax is the most prevalent human malaria parasite globally and is responsible for a large proportion of the global malaria burden, especially in regions outside of Africa [1]. Although it has been neglected as a benign infection, $P$. vivax causes serious clinical illnesses including respiratory distress, severe anemia, coma and even death [2, 3]. Moreover, it has recently re-emerged in many temperate regions from where it had been largely eradicated during global malaria control campaigns. The emergence of drug resistance strains also complicates the burden of the parasite $[4,5]$. Considering the enormous socio-economic impact of $P$. vivax on humans, development of an effective vaccine is an important concern in control and elimination strategies. However, no effective vaccine is yet available and the antigenic diversity present in wild-type isolates, which has led to the failure of several licensed and tested malaria vaccines, has been recognized as a major concern in developing a successful vaccine.

Apical membrane antigen-1 (AMA-1) is a type 1 integral membrane protein that is expressed in the late schizont stage of malaria parasites [6]. It is initially synthesized in the micronemes of the apical complex of merozoites and is transported to the surface of parasite just prior to erythrocyte invasion, where it undergoes proteolytic cleavage $[7,8]$. Although the precise role of AMA-1 is not fully understood, it is believed to be essential in erythrocyte invasion [9]. AMA-1 consists of a signal sequence, a cysteine-rich ectodomain, a conserved cytoplasmic region and a transmembrane region. The ectodomain is further divided into three distinct domains (domains I, II and III) by disulfide bridges [10]. The ectodomain of AMA-1 is very immunogenic and a high antibody titer against the domain is produced in humans who are naturally infected with malaria parasites [11-14]. Antibodies against AMA-1 effectively inhibit erythrocyte invasion [15-17]. Therefore, AMA-1 has been considered as a promising candidate antigen for blood stage malaria vaccine $[18,19]$.

Although it is recognized that AMA-1 is less variable than the other blood stage malaria vaccine candidate antigens such as merozoite surface protein antigens (MSPs) and circumsporozoite protein (CSP), it also shows sequence variations among global malaria parasites [20-23]. In P. falciparum, most of the genetic polymorphisms are concentrated in domain I [20, 21, 24], while the majority of the polymorphic patterns of AMA-1 occur in domains I and II in P. vivax [22-27]. These polymorphisms result in amino acid changes in the natural population, indicating that PvAMA-1 is under natural selection, may be a result of host immune pressure $[23,26]$.
In this study, the population genetic structure and natural selection of PvAMA-1 among Korean P. vivax isolates was analysed. A higher rate of polymorphic patterns and evidence of natural selection were identified in domains I and III. The Korean PvAMA-1 showed different polymorphic patterns compared to other global isolates. Recombination also likely has been important in generating genetic diversity across the PvAMA-1 sequences. These results provide useful information for the understanding of the population structure of $P$. vivax circulating in Korea and have important implications for the design of a vaccine incorporating PvAMA-1.

\section{Methods}

\section{Blood samples and study areas}

A total of 66 blood samples from Korean patients with uncomplicated $P$. vivax were collected at endemic areas between 2009 and 2011. The $P$. vivax infection was confirmed by microscopic examination of thin and thick blood smears and species-specific polymerase chain reaction (PCR) [28]. All the patients resided in malaria endemic regions, northwestern part of Gyeonggi province near DMZ in Korea, and have not been abroad at least in past 2 years when their blood samples were collected. The collected blood was separated into packed cells and plasma and then stored at $-80{ }^{\circ} \mathrm{C}$ until use. Informed consent was obtained from all of the patients before blood collection. The study protocol was approved by the Ethics Committee of the Inha University School of Medicine.

\section{Amplification and sequencing analysis of the PvAMA-1}

Genomic DNA of parasite was extracted from $200 \mu \mathrm{l}$ of whole blood sample using the QIAamp DNA Blood Kit (Qiagen, Hilden, Germany). The entire PvAMA-1 gene was amplified using two rounds of PCR. The primers were designed based on conserved regions of PvAMA-1 sequences of Sal I (AF063138) and Belem (EU395595). The forward and reverse primers used for the first round of PCR were 5'-GCAGAGAGAGCAAACC AAATCG-3' and 5'-GCAAGCGAGTTGGCCAAGC AAA- $3^{\prime}$. The primers used for the nested PCR were 5'-ATGAATAAAATATACTACATAATCTTTTTA- ${ }^{\prime}$ and 5'-TTAGTAGTATGGCTTCTCCATCAG-3'. The following thermal cycling conditions were used for both amplifications: $94{ }^{\circ} \mathrm{C}$ for $5 \mathrm{~min}$; 30 cycles of $94{ }^{\circ} \mathrm{C}$ for $1 \mathrm{~min}, 52{ }^{\circ} \mathrm{C}$ for $1 \mathrm{~min}$ and $72{ }^{\circ} \mathrm{C}$ for $2 \mathrm{~min}$, and a final extension at $72{ }^{\circ} \mathrm{C}$ for $10 \mathrm{~min}$. To minimize the nucleotide mis-incorporation in the sequences during PCR amplification, Ex Taq DNA polymerase (Takara, Otsu, Japan), which has a proofreading activity, was used in all PCR process. Each resulting PCR product was resolved on a $1.2 \%$ agarose gel, purified from the gel, and ligated into the T\&A cloning vector (Real Biotech Corporation, 
Banqiao City, Taiwan). Each ligation mixture was transformed into Escherichia coli DH5 $\alpha$ competent cells and positive clones with the appropriate insert were selected by colony PCR. The nucleotide sequences of the cloned insert were analysed by automatic DNA sequencing with M13 forward and M13 reverse primers. Sequencing analyses with two additional specific internal primers (5'-GAAGTTAAACGATATAGCTTTGTGCAG-3' and 5'-TCAACACTGTACAGATTCATGTTCCTC-3') were also performed to obtain the sequences of central region of PvAMA-1. At least two clones from each isolate were sequenced to ensure sequencing accuracy, and some isolates underwent three- or four-fold sequence coverage to confirm the existence of rare polymorphisms. The nucleotide sequences reported in this study have been deposited in the GenBank database under the accession numbers (KM230319-KM230384).

\section{Sequence and phylogenetic analysis of PvAMA-1}

The nucleotide and deduced amino acid sequences of PvAMA-1 were analysed using EditSeq and SeqMan in the DNASTAR package (DNASTAR, Madison, WI, USA). The phylogenetic tree was constructed using the neighbour-joining method in MEGA4 computational program [29]. Bootstrap proportions were used to assess the robustness of the tree with 1000 bootstrap replicates.

\section{Sequence analysis}

DNA sequence polymorphism analysis was performed on the 66 PvAMA-1 sequences. The number of segregating sites $(\mathrm{S})$, haplotypes $(\mathrm{H})$, haplotype diversity $(\mathrm{Hd})$, nucleotide diversity $(\pi)$, and the average number of pair-wise nucleotide differences within the population $(K)$ were estimated using the DnaSP ver. 5.10.00 [30]. The $\pi$ was calculated to estimate the step-wise diversity throughout the entire PvAMA-1 based on a sliding window of 100 bases with a step size of $25 \mathrm{bp}$. The rates of synonymous (dS) and non-synonymous (dN) substitutions were estimated and were compared using the $\mathrm{Z}$ test $(\mathrm{P}<0.05)$ in MEGA4 program [29] using Nei and Gojobori's method [31] with the Jukes and Cantor correction. To evaluate the neutral theory of evolution, the Tajima's $\mathrm{D}$ value [32] and Fu and Li's D and F statistics [33] were analysed using the DnaSP ver. 5.10.00 [30].

\section{Recombination parameters and linkage disequilibrium}

The recombination parameter $(\mathrm{R})$, which included the effective population size and probability of recombination between adjacent nucleotides per generation, and the minimum number of recombination events (Rm) were determined using the DnaSP ver. 5.10.00 [30]. The linkage disequilibrium (LD) between the different polymorphic sites was computed based on the $\mathrm{R}^{2}$ index.

\section{Results}

\section{Sequence polymorphism in Korean PvAMA-1}

Sixty-six PvAMA-1 sequences with 30 different haplotypes were obtained from Korean $P$. vivax isolates. Phylogenetic analysis of the sequences classified the 66 Korean PvAMA-1 sequences into seven distinct clusters (clusters A-G; Fig. 1). Nucleotide sequence analysis of the 66 sequences compared to SalI (AF063138) revealed 66 single nucleotide polymorphisms (SNPs) in the Korean PvAMA-1 sequences. The 22 SNPs were synonymous and the others were non-synonymous. The non-synonymous SNPs resulted in 44 dimorphic amino acid changes in 66 PvAMA-1 sequences (Fig. 2). Although these amino acid changes were not evenly distributed in each PvAMA-1 haplotype, four amino acid changes (D107A, K120R, N132D and E277K) were commonly found in all PvAMA-1 sequences (Fig. 2). L384P was commonly identified in the sequences in clusters A, B, C and D. E227V and S228D were highly conserved in sequences belonging to clusters B, C, D, E, F and G. T191K and N445D were also highly conserved in sequences in clusters $C, D$, E, F and $G$. The other amino acid changes were randomly distributed in each haplotype or were conserved among the sequences that were classified into the same cluster. For example, R112T was identified only in the sequences clustered into clusters A and B. Meanwhile, K86R, E189K, R471G and K401R were conserved in cluster A, $B, C$ and $E$, respectively.

These amino acid changes were mainly identified in domain I, but with significant rates in domains II and III. Six amino acid changes (Q25K, T31A, G491R, D520G, A540T and E545G) were found in the $5^{\prime}$-terminal $\left(5^{\prime}-\mathrm{T}\right)$ or $3^{\prime}$-terminal $\left(3^{\prime}-\mathrm{T}\right)$ region. Among the 44 dimorphic amino acid changes, 15 have been previously reported. The remaining 29 changes (Q25K, T31A, K61E, K86R, R88G, P90L, C94Y, V96A, F97L, H134R, T139A, M153T, L161V, T191K, C192R, R240C, I268V, R313G, V327M, K353R, C354R, K401R, D412G, I466T, R471G, G491R, D520G, A540T and E545G) are novel ones that have not been reported previously.

\section{Comparison of amino acid polymorphisms of Korean PvAMA-1 with global $P$. vivax isolates}

Korean PvAMA-1 showed different patterns of amino acid polymorphisms compared to those from other geographical regions (Fig. 3). The most significant characteristic in Korean PvAMA-1 was that four amino acid changes (D107A, K120R, N132D and E277K) were tightly conserved in all 66 PvAMA-1 sequences. E227V and S228D (63/66; $95.5 \%$, respectively), T191K and N445D (49/66; 74.2\%, respectively) and L384P (35/66; $53.03 \%)$ also showed high level of polymorphic patterns. High frequency of D107A was also identified in PvAMA-1 


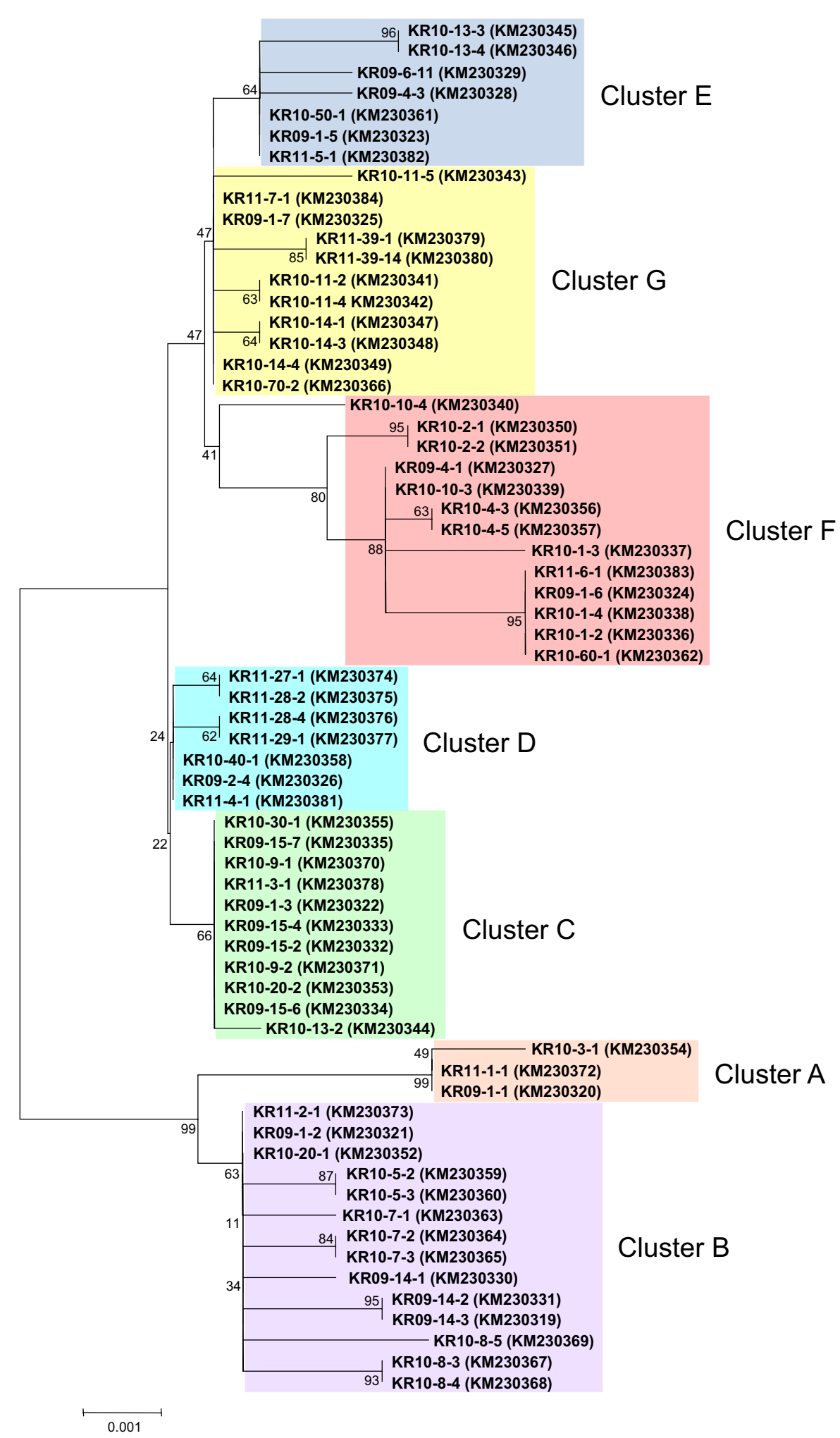

Fig. 1 Phylogenetic analysis of the PvAMA-1 sequences. The tree was constructed with 66 PvAMA-1 sequences obtained from Korean P. vivax isolates using a neighbour-joining method. The 66 sequences were divided into seven distinct clusters (cluster $A-G$ ). The tree was constructed using 1000 bootstrap replicates 


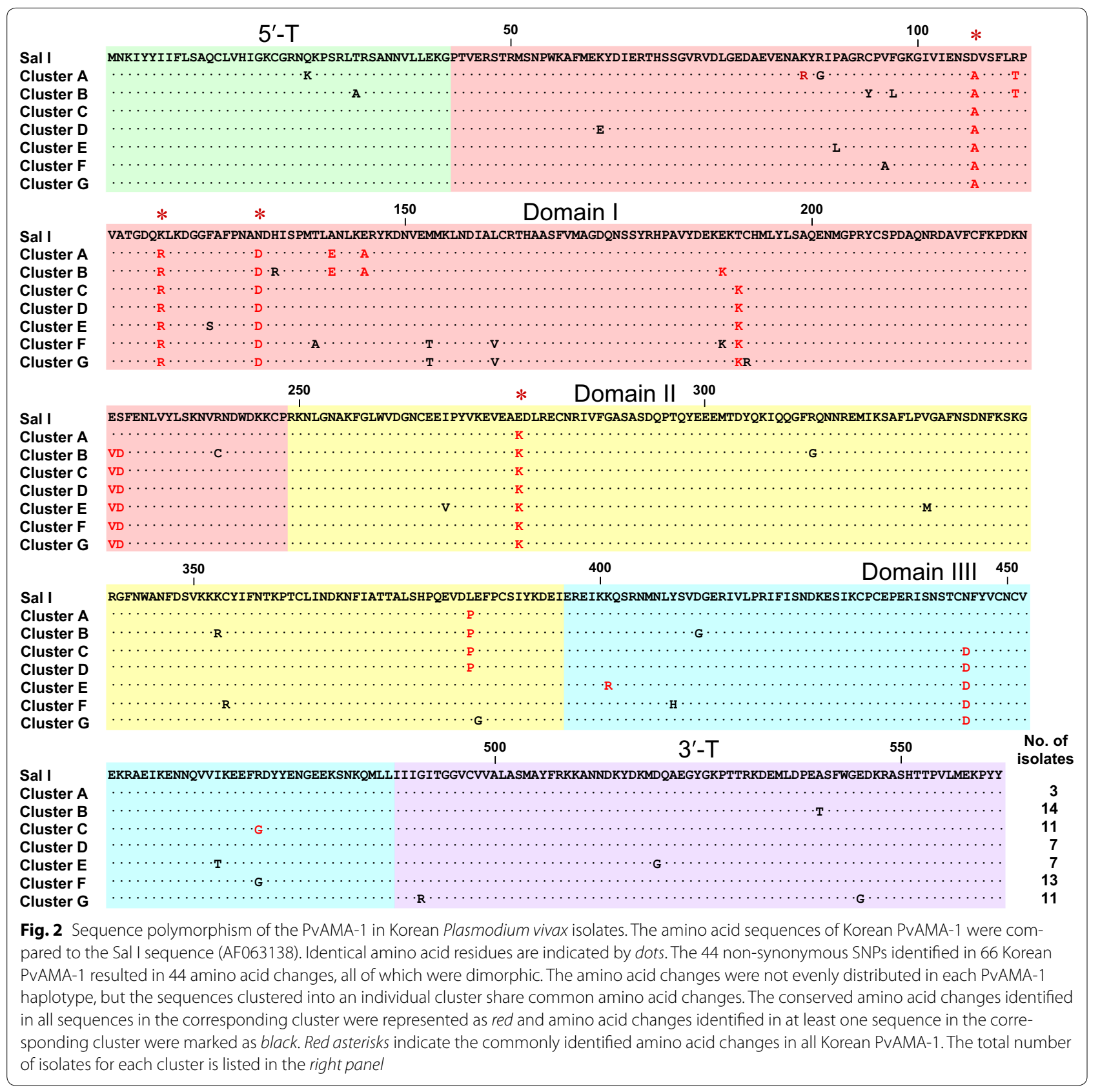

sequences from India (63.6\%), Thailand (72.2\%), Papua New Guinea (PNG, $73.5 \%$ ) and Venezuela (69.9\%), but its frequency was low in the sequences from Iran (29.7\%) and Sri Lanka (17.4 \%). K120R and E277K showed high level changes in the global PvAMA-1 sequences currently analysed. N132D was very frequent in India (81.8\%) and PNG (96.1\%), but was less than $50 \%$ in Iran, Sri Lanka, Thailand and Venezuela. E227V and S228D changes were tightly linked in Korean PvAMA-1 sequences. Interestingly, T191K and N445D, which were identified in high levels of frequency in Korean PvAMA-1, were not or rarely identified in PvAMA-1 sequences from other geographical areas. Meanwhile, R112K/T/S, L140I, E145A, P210S and $\mathrm{R} 438 \mathrm{H}$, which were highly polymorphic in PvAMA-1 sequences from other geographical areas, were not identified or detected at low levels in Korean PvAMA-1.

\section{Nucleotide diversity and natural selection}

DNA sequence analyses were conducted to determine nucleotide diversity and genetic differentiation 


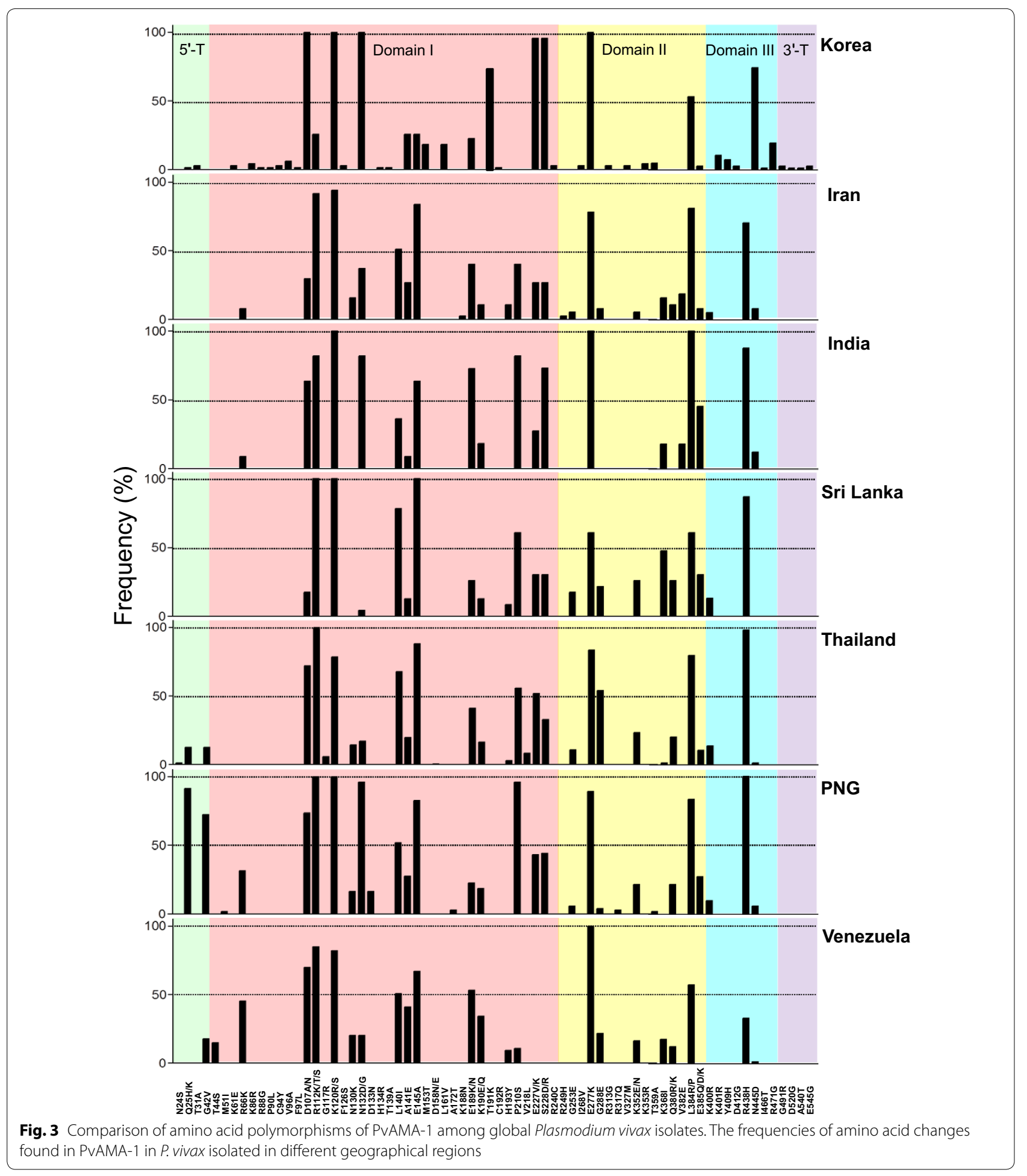

of PvAMA-1 in the Korean P. vivax isolates. The $K$ value for the $1687 \mathrm{bp}$ of full length PvAMA-1 was 8.07 (Table 1). The highest nucleotide differences was identified at domain I $(K=4.856)$ and was the lowest at $5^{\prime}-\mathrm{T}$.
The Hd for the entire PvAMA-1 sequences of 66 isolates was $0.961 \pm 0.012$. This value was higher in domain III than in domains I and II. The $\pi$ of the entire PvAMA-1 for the 66 Korean isolates was $0.00478 \pm 0.00038$. 


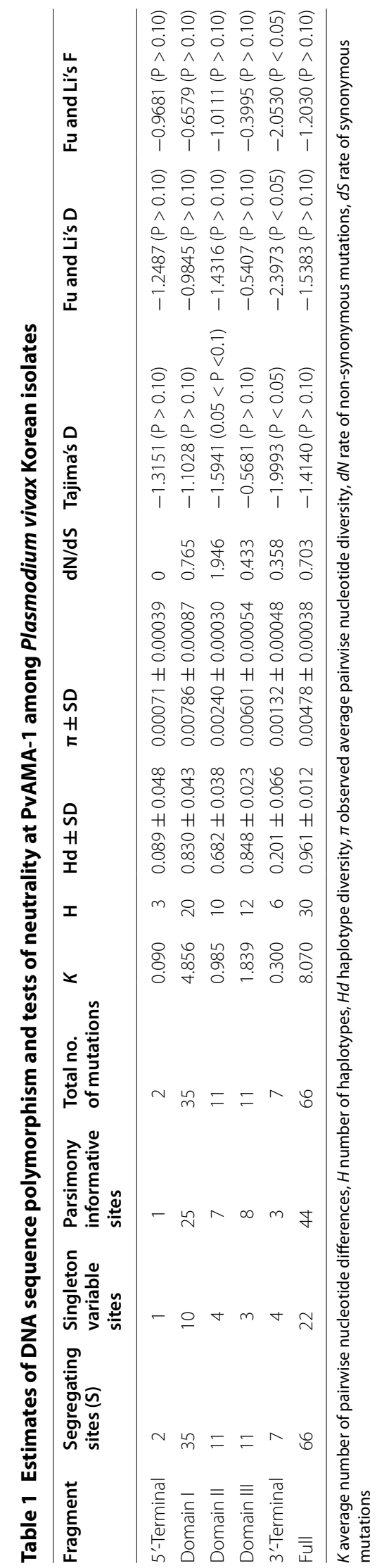


Analysis of the $\pi$ for the $5^{\prime}-T$, domain I, domain II, domain III and $3^{\prime}-\mathrm{T}$ indicated that most of the nucleotide diversity was concentrated in domains I and III. The $\pi$ value for each fragment was $0.00071 \pm 0.00039$ $\left(5^{\prime}-\mathrm{T}\right), 0.00786 \pm 0.00087$ (domain I), $0.00240 \pm 0.00030$ (domain II), $0.00601 \pm 0.00054$ (domain III) and $0.00132 \pm 0.00048\left(3^{\prime}-\mathrm{T}\right)$ (Table 1). Overall nucleotide diversity observed across the entire PvAMA-1 indicated peak nucleotide diversity in domains I and III (Fig. 4a). To examine whether natural selection contributed to generation of the diversity in PvAMA-1 of the Korean $P$. vivax population, the rate of $\mathrm{dN}$ to $\mathrm{dS}$ was estimated using the Nei and Gojobori's method [30]. The $\mathrm{dN}(0.00379)$ exceeded $\mathrm{dS}(0.00541)$, and the $\mathrm{dN} / \mathrm{dS}$ ratio of 0.703 suggested that negative natural selection may be occurring in the PvAMA-1 of Korean P. vivax isolates (Table 1). To further analyse the natural selection occurs in PvAMA-1 of Korean P. vivax population, Tajima's $\mathrm{D}$ test was performed. The estimated value was $-1.4140(\mathrm{P}>0.10)$, indicating that PvAMA-1 is under
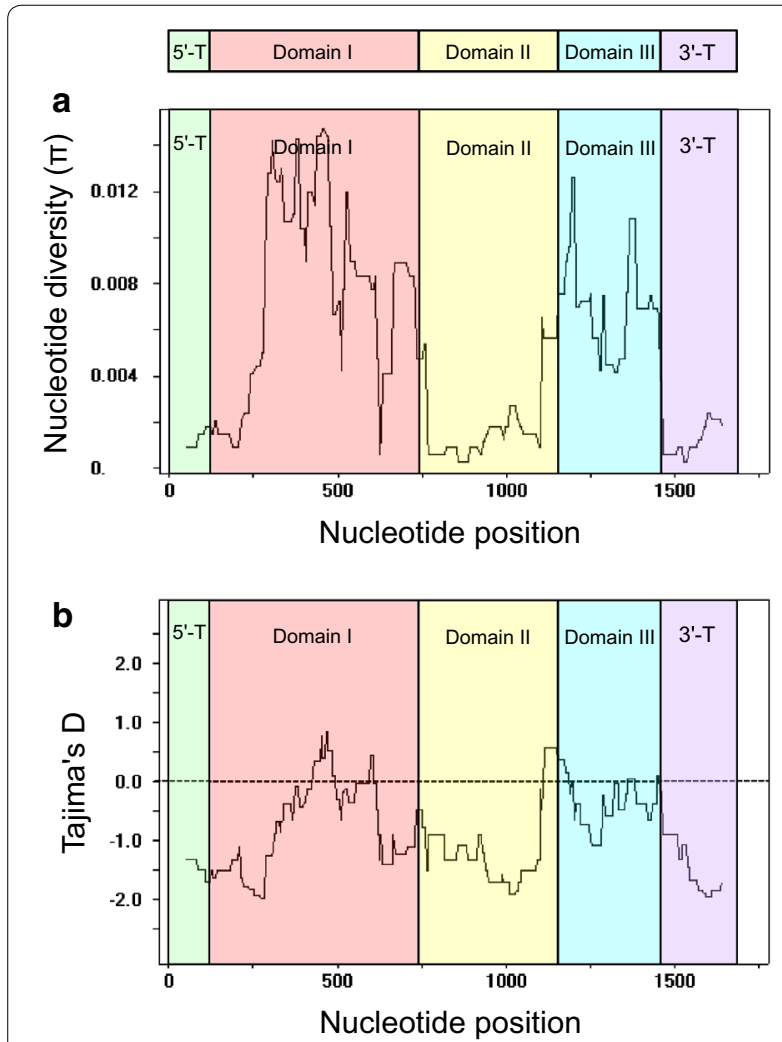

Fig. 4 Nucleotide diversity and natural selection of PVAMA-1 in Korean isolates. a Nucleotide diversity. Sliding window analysis showed nucleotide diversity $(\pi)$ values in 66 PvAMA-1 sequences analysed. A window size of $100 \mathrm{bp}$ and a step size of $25 \mathrm{bp}$ were used. b Natural selection. Sliding window calculation of Tajima's D statistic was performed for the 66 PvAMA-1 sequences. A window size of 100 and a step size of 25 were used population size expansion and/or purifying selection pressure (Table 1). The Fu and Li's D and F values for the entire PvAMA-1 were also negative: $-1.5383(\mathrm{P}>0.10)$ and $-1.2030(\mathrm{P}>0.10)$, respectively (Table 1$)$. Tajima's $\mathrm{D}$ values for each fragment of PvAMA-1 also showed negative values: $5^{\prime}-\mathrm{T}(-1.3151, \mathrm{P}>0.10)$, domain $\mathrm{I}(-1.1028$, $\mathrm{P}>0.10)$, domain II $(-1.5941,0.05<\mathrm{P}<0.10)$, domain III $(-0.5681, \mathrm{P}>0.10)$ and 3 '-T $(-1.9993, \mathrm{P}<0.05)$. However, the middle region of domain I and the junction between domains II and III showed positive Tajima's $\mathrm{D}$ values (Fig. 4b), indicating that the regions could be a dominant target of host immune response.

\section{Recombination and linkage disequilibrium}

For Korean PvAMA-1, the minimum number of recombination events between adjacent polymorphic sites ( $\mathrm{Rm})$ was estimated as 3 , whereas the $R$ between adjacent site (Ra) and per gene $(\mathrm{Rb})$ was 0.0056 and 9.5 , respectively. The plausible recombination sites were predicted to be localized in domain I and domain III. These high values of the recombination parameters suggested that meiotic recombination may occur between sites, resulting in genetic diversity of PvAMA-1. The LD index $\left(\mathrm{R}^{2}\right)$ also declined across PvAMA-1, suggesting that intragenic recombination may also be contributing the PvAMA-1 diversity (Fig. 5).

\section{Discussion}

Vivax malaria re-emerged in Korea in 1993 near the demilitarized zone (DMZ) border with North Korea [34]. Since its re-emergence, the outbreak has spread into cities and counties adjacent to the DMZ and has persisted until now with fluctuating numbers of annual indigenous cases, with 32,300 official cases [35]. Understanding the

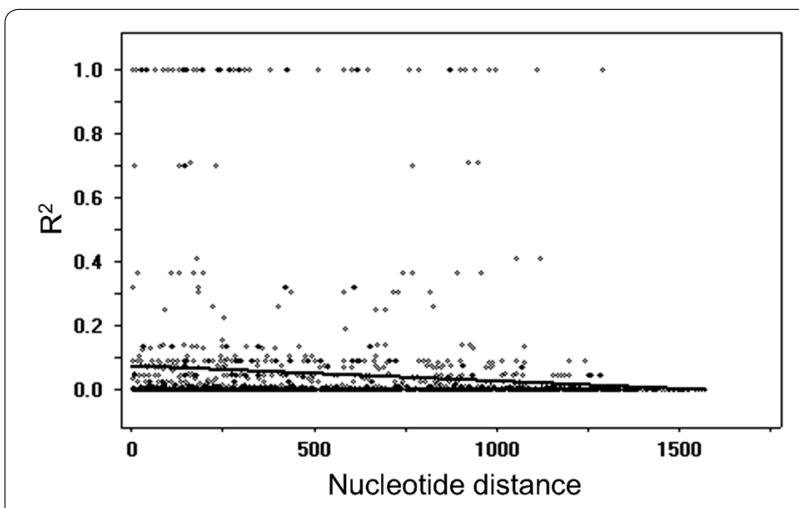

Fig. 5 Recombination event in PVAMA-1. The linkage disequilibrium (LD) plot showed non-random associations between the nucleotide variants in 66 Korean PvAMA-1 sequences at different polymorphic sites. The $R^{2}$ values were plotted against nucleotide distance using a two-tailed Fisher's exact test for statistical significance 
nature of the genetic population of $P$. vivax circulating in Korea is beneficial to knowledge of the nationwide parasite heterogeneity and in the implementation malaria control programs in the country. Moreover, this information is helpful for the development of an effective malaria vaccine. Several studies on genetic diversity of polymorphic marker proteins including MSP-1, MSP- $3 \alpha$, MSP- $3 \beta$ and CSP in Korean $P$. vivax suggested that rapid dissemination of genetic structure of $P$. vivax is processed in recent years [36-42]. Characterization of genetic property of PvAMA-1 in Korean P. vivax isolates has also been analysed [43, 44], but these studies analysed sequence polymorphism restricted to partial region corresponding domain I and not the entire PvAMA-1. In the present study, the genetic polymorphisms and natural selection in the entire PvAMA-1 among $P$. vivax Korean isolates were analysed to gain an in-depth understanding of the nature of PvAMA-1 in Korean P. vivax population.

The 66 PvAMA-1 sequences obtained from Korean $P$. vivax isolates were classified into 30 different haplotypes, which were further clustered into seven distinct clusters. Compared to Sal I sequence, 66 SNPs, which resulted in 44 dimorphic amino acid substitutions, were identified in Korean PvAMA-1. The majority of the amino acid changes clustered within PvAMA-1 domain I, as has been reported previously for $P$. vivax and $P$. falciparum AMA-1 [20-23, 26, 27, 45]. The 15 amino acid changes identified in Korean PvAMA-1 are comparable to those previously identified [22, 23, 26, 27], which suggests that the polymorphic sites are under high natural selection. The 29 amino acid changes were novel and have not been reported, even though they were detected with low frequencies in Korean PvAMA-1. Compared to previously reported North Korea (EU395599) and South Korea (SK0814; GU476844) sequences, which are only two entire PvAMA-1 sequences currently available in Korean Peninsula-origin $P$. vivax, Korean PvAMA-1 sequences analysed in this study shared similar patterns of amino acid changes. All amino acid changes reported in the North Korea and South Korea sequences were commonly identified in all Korean PvAMA-1 sequences analysed currently. In particular, the four tightly conserved amino acid changes (D107A, K120R, N132D and E277K), which are the most outstanding characteristic found in Korean PvAMA-1 analysed in this study, were well-conserved in the all the sequences. Based on the sequence polymorphism analyses of the current study, the North Korea sequence was most similar to the sequences belongs to cluster A, while the South Korea sequence was more closely related with the sequences of cluster D or G. Interestingly, Korean PvAMA-1 showed different patterns of polymorphism compared to previously reported sequences from other geographical regions
[22-27]. Although K120R/S and E277K were also identified at high frequencies (up to $50 \%$ ) in PvAMA-1 from other different geographical areas, D107A/N frequency was low in Iran (29.7 \%) and Sri Lanka (17.4 \%) and the N132D/G frequency was low in Iran (37.0\%), Sri Lanka (4.3\%), Thailand (17.1\%) and Venezuela (20.6\%) [2227]. S228D, which showed a high frequency $(95.5 \%)$ in Korean PvAMA-1, was identified with a high frequency only in Indian isolates (72.8\%) [25]. Meanwhile, the amino acid changes (R112K/T/S, L140I, E145A, P201S and $\mathrm{R} 438 \mathrm{H}$ ), which showed high frequencies in other geographical regions, were not identified or detected only with low levels of frequency in Korean PvAMA-1. Unlike the relatively even global distribution of PfAMA-1 diversity [46, 47], substantial geographical differentiation between populations was observed for PvAMA-1 [23]. The results of this study also suggest that Korean PvAMA-1 showed different patterns of polymorphic nature compared to those of other geographical areas and substantial geographic differentiation was observed among global isolates.

Several studies have suggested that the most common polymorphic amino acid residues identified in PvAMA-1 are located on one face of the protein, which suggests that this face is more exposed to the exterior environment and is accessible to host immune responses [21, 23, $27,48]$. Consistent with the previous studies, the majority of SNPs detected in Korean PvAMA-1 are exposed on one face of the protein. A recent study on the epitope mapping prediction of PvAMA-1 suggested that the potential B cell epitopes across the ectodomain [27]. Some of the SNPs identified in global P. vivax isolates, including E145K, P210S, R249H, G253E, K352E, R438H and N445D, overlap with the B-cell epitope regions. These amino acid changes may affect the protein structure by causing changes in charge and polarity of the protein and might help parasites to escape from host protective immunity [27]. No or few changes were observed in the corresponding amino acids in Korean PvAMA-1, but N445D was identified with a high frequency $(74.2 \%)$, which differs significantly from isolates from other different geographical areas. Three additional amino acid substitutions (Q380K, V382E and L384P/R) were also may alter protein structure by causing changes in protein polarity and hydrophilicity [27], which might decrease epitope binding scores or loss of the predicted linear B cell epitope either together or in combination with proximal polymorphisms [49]. Q380K and V382E were not identified in Korean PvAMA-1, but L384P was observed at a high frequency (53.3\%). Intrinsically unstructured/ disordered regions (IURs), which are widely identified in eukaryotic proteins, play important roles in many fundamental cell functions such as molecular recognition, 
molecular assembly and protein modification [50, 51]. Plasmodium spp. also have many proteins containing IURs and these proteins have been considered important in attachment and invasion of the parasite into red blood cells, although their specific functions remain to be defined [52]. Thereby overlap region of B-cell epitopes and IURs has been postulated more likely to represent a real antigenic/immunogenic region within a protein and the co-occurrence of both regions increases the chance of presenting this region with no secondary structure $[52,53]$. The predicted overlap IUR and B-cell epitope, which comprises 18 amino acid residues (SASDQPTQYEEEMTDYQK), is highly antigenic during natural human infections and is an important antigenic region of the domain II of PvAMA-1 [27, 53]. This linear epitope was highly conserved in all Korean PvAMA-1 sequences, as it was strictly conserved in currently analysed global isolates [27]. This suggests a high degree of amino acid sequence conservation of the region among global $P$. vivax isolates and further supports the hypothesis that these amino acid sequences in domain II are subjected to strong purifying selection that might be used as a component of a PvAMA-1-based vaccine.

The level of nucleotide diversity at entire PvAMA-1 in Korean $P$. vivax isolates $(\pi=0.00478)$ was relatively lower than those for isolates from different geographical areas, including Iran $(\pi=0.00826)$, Sri Lanka $(\pi=0.00675)$, PNG $(\pi=0.0079)$, Venezuela $(\pi=0.0065)$ and Thailand $(\pi=0.0089)[22,23,27]$. Lower malaria transmission in Korea compared to other endemic countries may contribute to the lower genetic diversity observed in Korean PvAMA-1. These collective results are suggestive of a low level of genetic polymorphism occurring at Korean PvAMA-1. The nucleotide diversity was not evenly distributed across entire PvAMA-1. The $5^{\prime}-\mathrm{T}$ and $3^{\prime}-\mathrm{T}$ showed low levels of nucleotide diversity, which suggests that the two regions are relatively well conserved in Korean PvAMA-1. A high level of nucleotide diversity was observed in domain I and domain III in PvAMA-1 of Korean P. vivax isolates, different from previous reports that nucleotide diversity is highest in domains I and II of PvAMA-1 in Indian, Sri Lankan and Thailand $P$. vivax isolates $[22,26,27]$. This indicates that domain II is more conservative with lower frequency polymorphisms than domains I and III in Korean PvAMA-1, even though a cluster of SNPs was observed in domain II. Natural selection affected by host immune responses and recombination between genetically distinct malaria parasites during meiotic replication in the mosquito midgut have been recognized as the two main mechanisms by which PvAMA-1 genetic diversity is generated and maintained [49]. The dN/dS for the entire length Korean PvAMA-1 was estimated to 0.703 , suggesting that purifying selection pressure may act on the protein [54]. However, the $\mathrm{dN} / \mathrm{dS}$ value of domain II was higher than 1 (1.946), which implies that positive natural selection may be occurring in the domain. Negative values of Tajima's $\mathrm{D}(-1.4140), \mathrm{Fu}$ and Li's D $(-1.5383)$ and Fu and Li's F $(-1.2030)$ for entire Korean PvAMA-1 presently analysed imply an excess of low frequency polymorphisms indicating population size expansion and/or purifying selection [31]. Each domain of Korean PvAMA-1 also showed negative values of Tajima's D, Fu and Li's D and Fu and Li's $F$. These results clearly point to stronger diversifying selection, probably by host immune selection pressure, is working at Korean PvAMA-1. Meiotic recombination that occurs between the adjacent polymorphic sites drives allelic diversity of PvAMA-1 [22, 26]. Evidence of recombination event within the Korean PvAMA-1 was also observed, which supported by the decline of LD index $\mathrm{R}^{2}$ with increasing nucleotide distance, as consistent with the previous studies.

\section{Conclusions}

A major concern in the development of effective malaria vaccine is genetic polymorphisms observed among global isolates. PvAMA-1 is one of the most promising candidates for malaria vaccine targeting the blood stages of $P$. vivax. Genetic investigation of Korean PvAMA-1 suggests that this antigen shows a limited antigenic diversity, but most of the major amino acid polymorphisms located on one face of the protein were commonly identified among worldwide $P$. vivax isolates. However, the overall pattern of genetic polymorphism of Korean PvAMA-1 slightly differed from those from other geographical isolates. Moreover, 29 novel polymorphic changes, most of which are predicted to be located outside B-cell epitopes, were also observed in Korean PvAMA-1. Genetic diversity of Korean PvAMA-1 was relatively lower than currently analyzed PvAMA-1 from other geographical areas, but evidences for natural selection and recombination were found. These results have significant implications in understanding the nature of Korean $P$. vivax population and in providing useful information for malaria vaccine development based on this antigen.

\section{Authors' contributions}

$J M K$, JL and $H \sqcup$ performed all the experiments and analysed the sequence data. PYC, SKA and SUM collected blood samples or performed sequence and phylogenetic analyses. BKN and TSK designed the study and supervised the study process. JMK, JL and BKN wrote the paper. TSK, HWL and WMS assisted in writing and editing the manuscript. All authors read and approved the final manuscript.

\section{Author details}

${ }^{1}$ Department of Parasitology and Tropical Medicine, Institute of Health Sciences, Gyeongsang National University School of Medicine, Jinju 660-751, Republic of Korea. ${ }^{2}$ Department of Tropical Medicine, Inha Research Institute 
for Medical Sciences, Inha University School of Medicine, Incheon 400-712, Republic of Korea. ${ }^{3}$ Department of Internal Medicine, Seoul National University Bundang Hospital, Seongnam 463-707, Republic of Korea. ${ }^{4}$ Department of Pathology, Immunology, and Laboratory Medicine, College of Medicine, University of Florida, J-566, 1275 Center Drive, Gainesville, FL 32610, USA.

\section{Acknowledgements}

This work was supported by the National Research Foundation of Korea (NRF) grants funded by the Korea government (MEST) (2011-0028135) and by the Korea government (MSIP) (2015R1A2A2A01004310).

\section{Competing interests}

The authors declare that they have no competing interests.

Received: 18 May 2015 Accepted: 9 October 2015

Published online: 16 November 2015

\section{References}

1. Baird JK. Neglect of Plasmodium vivax malaria. Trends Parasitol. 2007:23:533-9.

2. Rogerson SJ, Carter R. Severe vivax malaria: newly recognised or rediscovered. PLoS Med. 2008;5:e136.

3. Anstey NM, Russell B, Yeo TW, Price RN. The pathophysiology of vivax malaria. Trends Parasitol. 2009;25:220-7.

4. Baird JK. Chloroquine resistance in Plasmodium vivax. Antimicrob Agents Chemother. 2004;48:4075-83.

5. Price RN, Tjitra E, Guerra CA, Yeung S, White NJ, Anstey NM. Vivax malaria: neglected and not benign. Am J Trop Med Hyg. 2007;77:79-87.

6. Peterson MG, Marshall VM, Smythe JA, Crewther PE, Lew A, Silva A, et al. Integral membrane protein located in the apical complex of Plasmodium falciparum. Mol Cell Biol. 1989:9:3151-4.

7. Bannister LH, Hopkins JM, Dluzewski AR, Margos G, Williams IT, Blackman MJ, et al. Plasmodium falciparum apical membrane antigen 1 (PfAMA-1) is translocated within micronemes along subpellicular microtubules during merozoite development. J Cell Sci. 2003;116:3825-34.

8. Howell SA, Well I, Fleck SL, Kettleborough C, Collins CR, Blackman MJ. A single malaria merozoite serine protease mediates shedding of multiple surface proteins by juxtamembrane cleavage. J Biol Chem. 2003;26:23890-8.

9. Hodder AN, Crewther PE, Anders RF. Specificity of the protective antibody response to apical membrane antigen 1. Infect Immun. 2001;69:3286-94.

10. Hodder AN, Crewther PE, Matthew ML, Reid GE, Moritz RL, Simpson RJ, et al. The disulfide bond structure of Plasmodium apical membrane antigen-1. J Biol Chem. 1996;271:29446-52.

11. Udhayakumar V, Kariuki S, Kolczack M, Girma M, Roberts JM, Oloo AJ, et al. Longitudinal study of natural immune responses to the Plasmodium falciparum apical membrane antigen (AMA-1) in a holoendemic region of malaria in western Kenya: Asembo Bay Cohort Project VIII. Am J Trop Med Hyg. 2001;65:100-7.

12. Polley SD, Mwangi T, Kocken CH, Thomas AW, Dutta S, Lanar DE, et al. Human antibodies to recombinant protein constructs of Plasmodium falciparum apical membrane antigen 1 (AMA1) and their associations with protection from malaria. Vaccine. 2004;23:718-28.

13. Múfalo BC, Gentil F, Bargieri DY, Costa FT, Rodrigues MM, Soares IS. Plasmodium vivax apical membrane antigen-1: comparative recognition of different domains by antibodies induced during natural human infection. Microbes Infect. 2008;10:1266-73.

14. Gentil F, Bargieri DY, Leite JA, Françoso KS, Patricio MB, Espíndola NM, Vaz AJ, et al. A recombinant vaccine based on domain II of Plasmodium vivax apical membrane antigen 1 induces high antibody titres in mice. Vaccine. 2010;28:6183-90.

15. Kocken $\mathrm{CH}$, Withers-Martinez C, Dubbeld MA, van der Wel A, Hackett F, Valderrama A, et al. High-level expression of the malaria blood-stage vaccine candidate Plasmodium falciparum apical membrane antigen 1 and induction of antibodies that inhibit erythrocyte invasion. Infect Immun. 2002;70:4471-6.

16. Dutta S, Haynes JD, Moch JK, Barbosa A, Lanar DE. Invasion-inhibitory antibodies inhibit proteolytic processing of apical membrane antigen 1 of Plasmodium falciparum merozoites. Proc Natl Acad Sci USA. 2003;100:12295-300.
17. Woehlbier U, Epp C, Hackett F, Blackman MJ, Bujard H. Antibodies against multiple merozoite surface antigens of the human malaria parasite Plasmodium falciparum inhibit parasite maturation and red blood cell invasion. Malar J. 2010;9:77.

18. Mitchell GH, Thomas AW, Margos G, Dluzewski AR, Bannister LH. Apical membrane antigen 1, a major malaria vaccine candidate, mediates the close attachment of invasive merozoites to host red blood cells. Infect Immun. 2004;72:154-8.

19. Remarque EJ, Faber BW, Kocken $\mathrm{CH}$, Thomas AW. Apical membrane antigen 1: a malaria vaccine candidate in review. Trends Parasitol. 2008;24:74-84.

20. Escalante AA, Grebert HM, Chaiyaroj SC, Magris M, Biswas S, Nahlen BL, et al. Polymorphism in the gene encoding the apical membrane antigen-1 (AMA-1) of Plasmodium falciparum. X. Asembo Bay Cohort Project. Mol Biochem Parasitol. 2001;113:279-87.

21. Polley SD, Conway DJ. Diversifying selection on domains of the Plasmodium falciparum apical membrane antigen 1 gene. Genetics. 2001;158:1505-12.

22. Gunasekera AM, Wickramarachchi T, Neafsey DE, Ganguli I, Perera L, Premaratne $\mathrm{PH}$, et al. Genetic diversity and selection at the Plasmodium vivax apical membrane antigen-1 (PvAMA-1) locus in a Sri Lankan population. Mol Biol Evol. 2007;24:939-47.

23. Arnott A, Mueller I, Ramsland PA, Siba PM, Reeder JC, Barry AE. Global population structure of the genes encoding the malaria vaccine candidate, Plasmodium vivax apical membrane antigen 1(PvAMA1). PLoS Negl Trop Dis. 2013;7:e2506.

24. Ord RL, Tami A, Sutherland CJ. Ama1 genes of sympatric Plasmodium vivax and $P$. falciparum from Venezuela differ significantly in genetic diversity and recombination frequency. PLoS One. 2008:3:e3366.

25. Rajesh V, Elamaran M, Vidya S, Gowrishankar M, Kochar D, Das A. Plasmodium vivax: genetic diversity of the apical membrane antigen-1 (AMA-1) in isolates from India. Exp Parasitol. 2007;116:252-6.

26. Putaporntip C, Jongwutiwes S, Grynberg P, Cui L, Hughes AL. Nucleotide sequence polymorphism at the apical membrane antigen-1 locus reveals population history of Plasmodium vivax in Thailand. Infect Genet Evol. 2009;9:1295-300

27. Zakeri S, Sadeghi H, Mehrizi AA, Djadid ND. Population genetic structure and polymorphism analysis of gene encoding apical membrane antigen-1 (AMA1) of Iranian Plasmodium vivax wild isolates. Acta Trop. 2013;126:269-79.

28. Snounou G, Viriyakosol S, Jarra W, Thaithong S, Brown KN. Identification of the four human malaria parasite species in field samples by the polymerase chain reaction and detection of a high prevalence of mixed infections. Mol Biochem Parasitol. 1993;58:283-92.

29. Tamura K, Dudley J, Nei M, Kumar S. MEGA4: molecular evolutionary genetics analysis (MEGA) software version 4.0. Mol Biol Evol. 2007;24:1596-9.

30. Librado P, Rozas J. DnaSP v5: a software for comprehensive analysis of DNA polymorphism data. Bioinformatics. 2009;25:1451-2.

31. Nei M, Gojobori T. Simple methods for estimating the numbers of synonymous and nonsynonymous nucleotide substitutions. Mol Biol Evol. 1986:3:418-26.

32. Tajima F. Statistical method for testing the neutral mutation hypothesis by DNA polymorphism. Genetics. 1989;123:585-95.

33. Fu YX, Li WH. Statistical tests of neutrality of mutations. Genetics. 1993;133:693-709.

34. Chai IH, Lim Gl, Yoon SN, Oh WI, Kim SJ, Chai JY. Occurrence of tertian malaria in a male patient who has never been abroad. Korean J Parasitol. 1994;32:195-200

35. Kim TS, Kim JS, Na BK, Lee WJ, Kim HC, Youn SK, et al. Decreasing incidence of Plasmodium vivax in the Republic of Korea during 2010-2012. Malar J. 2013;12:309.

36. Choi YK, Choi KM, Park MH, Lee EG, Kim YJ, Lee BC, et al. Rapid dissemination of newly introduced Plasmodium vivax genotypes in South Korea. Am J Trop Med Hyg. 2010;82:426-32.

37. Honma H, Kim JY, Palacpac NM, Mita T, Lee W, Horii T, et al. Recent increase of genetic diversity in Plasmodium vivax population in the Republic of Korea. Malar J. 2011;10:257.

38. Kang JM, Ju HL, Kang YM, Lee DH, Moon SU, Sohn WM, et al. Genetic polymorphism and natural selection in the C-terminal $42 \mathrm{kDa}$ region of merozoite surface protein-1 among Plasmodium vivax Korean isolates. Malar J. 2012:11:206. 
39. Iwagami M, Hwang SY, Kim SH, Park SJ, Lee GY, Matsumoto-Takahashi EL, et al. Microsatellite DNA analysis revealed a drastic genetic change of Plasmodium vivax population in the Republic of Korea during 2002 and 2003. PLoS Negl Trop Dis. 2013;7:e2522.

40. Ju HL, Kang JM, Moon SU, Bahk YY, Cho PY, Sohn WM, et al. Genetic diversity and natural selection of Duffy binding protein of Plasmodium vivax Korean isolates. Acta Trop. 2013;125:67-74.

41. Kang JM, Ju HL, Cho PY, Moon SU, Ahn SK, Sohn WM, et al. Polymorphic patterns of the merozoite surface protein-3 $\beta$ in Korean isolates of Plasmodium vivax. Malar J. 2014;13:104.

42. Han ET, Song TE, Park JH, Shin EH, Guk SM, Kim TY, et al. Allelic dimorphism in the merozoite surface protein-3alpha in Korean isolates of Plasmodium vivax. Am J Trop Med Hyg. 2004;71:745-9.

43. Han ET, Park JH, Shin EH, Choi MH, Oh MD, Chai JY. Apical membrane antigen-1 (AMA-1) gene sequences of re-emerging Plasmodium vivax in South Korea. Korean J Parasitol. 2002;40:157-62.

44. Chung JY, Chun EH, Chun JH, Kho WG. Analysis of the Plasmodium vivax apical membrane antigen-1 gene from re-emerging Korean isolates. Parasitol Res. 2003;90:325-9.

45. Moon SU, Na BK, Kang JM, Kim JY, Cho SH, Park YK, et al. Genetic polymorphism and effect of natural selection at domain I of apical membrane antigen-1 (AMA-1) in Plasmodium vivax isolates from Myanmar. Acta Trop. 2010;114:71-5.

46. Duan J, Mu J, Thera MA, Joy D, Kosakovsky Pond SL, Diemert D, et al. Populationstructure of the genes encoding the polymorphic Plasmodium falciparum apical membrane antigen 1: implications for vaccine design. Proc Natl Acad Sci USA. 2008;105:7857-62.
47. Barry AE, Schultz L, Buckee CO, Reeder JC. Contrasting population structures of the genes encoding ten leading vaccine-candidate antigens of the human malaria parasite, Plasmodium falciparum. PLoS One. 2009;4:e8497.

48. Polley SD, Chokejindachai W, Conway DJ. Allele frequency-based analyses robustly map sequence sites under balancing selection in a malaria vaccine candidate antigen. Genetics. 2003;165:555-61.

49. Dias S, Somarathna M, Manamperi A, Escalante AA, Gunasekera AM, Udagama PV. Evaluation of the genetic diversity of domain II of Plasmodium vivax apical membrane antigen 1 (PVAMA-1) and the ensuing strain-specific immune responses in patients from Sri Lanka. Vaccine. 2011;29:7491-504.

50. Dunker AK, Brown CJ, Lawson JD, lakoucheva LM, Obradovic Z. Intrinsic disorder and protein function. Biochemistry. 2002;41:6573-82.

51. Dyson HJ, Wright PE. Intrinsically unstructured proteins and their functions. Nat Rev Mol Cell Biol. 2005;6:197-208.

52. Feng ZP, Zhang X, Han P, Arora N, Anders RF, Norton RS. Abundance of intrinsically unstructured proteins in P. falciparum and other apicomplexan parasite proteomes. Mol Biochem Parasitol. 2006;150:256-67.

53. Bueno LL, Lobo FP, Morais CG, Mourão LC, de Ávila RA, Soares IS, et al. Identification of a highly antigenic linear B cell epitope within Plasmodium vivax apical membrane antigen 1 (AMA-1). PLoS One. 2011;6:e21289.

54. Escalante AA, Cornejo OE, Rojas A, Udhayakumar V, Lal AA. Assessing the effect of natural selection in malaria parasites. Trends Parasitol. 2004;20:388-95.

\section{Submit your next manuscript to BioMed Central and take full advantage of:}

- Convenient online submission

- Thorough peer review

- No space constraints or color figure charges

- Immediate publication on acceptance

- Inclusion in PubMed, CAS, Scopus and Google Scholar

- Research which is freely available for redistribution

Submit your manuscript at

www.biomedcentral.com/submit

C Biomed Central 